\title{
Screening and Prognostic Value of Methylated Septin9 and its Association With Clinicopathological and Molecular Characteristics in Colorectal Cancer
}

\author{
Jie Sun ${ }^{1 \dagger}$, Jinling $\mathrm{Xu}^{1 \dagger}$, Chao Sun ${ }^{2 \dagger}$, Minying Zheng ${ }^{1}$, Yuwei $\mathrm{Li}^{3}$, Siwei Zhu ${ }^{1}$ and \\ Shiwu Zhang ${ }^{1 *}$
}

${ }^{1}$ Department of Pathology, Tianjin Union Medical Center, Tianjin, China, ${ }^{2}$ Graduate School, Tianjin University of Traditional Chinese Medicine, Tianjin, China, ${ }^{3}$ Department of Colorectal Surgery, Tianjin Union Medical Center, Tianjin, China

\section{OPEN ACCESS}

Edited by:

Anton A. Buzdin,

I. M. Sechenov First Moscow State Medical University, Russia

Reviewed by:

Yoshikatsu Koga,

National Cancer Centre, Japan Marianna Arsenovna Zolotovskaia, Pirogov Russian National Research Medical University, Russia

*Correspondence: Shiwu Zhang zhangshiwu666@aliyun.com

${ }^{+}$These authors have contributed equally to this work

Specialty section:

This article was submitted to Molecular Diagnostics and Therapeutics,

a section of the journal Frontiers in Molecular Biosciences

Received: 24 July 2020 Accepted: 22 April 2021

Published: 20 May 2021

Citation:

Sun J, Xu J, Sun C, Zheng M, Li Y, Zhu S and Zhang S (2021) Screening and Prognostic Value of Methylated Septing and its Association With Clinicopathological and Molecular Characteristics in Colorectal Cancer. Front. Mol. Biosci. 8:568818 doi: 10.3389/fmolb.2021.568818
Screening of CRC continues to show poor compliance of endoscopy examination. The detection of mSEPT9 in peripheral blood is among the safe and simple early screening methods for CRC. The issue of how to elucidate whether detection of mSEPT9 in peripheral blood can effectively improve compliance of endoscopy and increase the early diagnosis rate of $\mathrm{CRC}$ and the relationship between levels of mSEPT9 in the peripheral blood and clinical stage, pathological classification, and expression of characteristic molecules in CRC remains unsolved. A total of 7759 individuals participated in the study that was performed using a questionnaire for screening of high-risk CRC. The endoscopic detection compliance of individuals with high-risk CRC who underwent the fecal occult blood test (FOBT) or mSEPT9 test was compared based on the results of the questionnaire. Additionally, correlation of mSEPT9 levels in the peripheral blood with clinicopathological features, mutation status of TP53, mismatch repair deficiency (dMMR), and KRAS/NRAS/BRAF/ PIK3CA genotype was analyzed, and association of biomarkers with cancer-specific survival (CSS) and time to recurrence (TTR) was compared. We also detected levels of mSEPT9 in the peripheral blood of patients with CRC 7 days after surgery and compared the prognostic value of mSEPT9 with CEA. Results of our study showed that the mSEPT9 test could improve compliance of endoscopy and indicated a higher percentage of patients with positive mSEPT9 willing to undergo endoscopy detection than in those with positive FOBT. The specificity and sensitivity of mSEPT9 were better than that of FOBT for the detection of CRC. mSEPT9 was associated with the TNM stage, dMMR, and mutations in TP53, BRAF, and PIK3CA. A Ct value of mSEPT9 $\leq 37.5$ was significantly related to poor CSS. mSEPT9 could affect association of dMMR and BRAF and PIK3CA mutations with CSS in a specific stage of CRC. The positive rate of $\mathrm{MSEPT9}$ after surgery was found to correlate with poor TTR, and sensitivity was higher than CEA. The combination of mSEPT9 with CEA had a better

Abbreviations: CEA, carcinoembryonic antigen; CIMP, CpG island methylator phenotype; CIN, chromosome instability; CRC, colorectal cancer; Ct, cycle threshold; dMMR, mismatch repair deficiency; FOBT, fecal occult blood test; mSEPT9, methylated Septin9; MSI, microsatellite instability; CSS, cancer-specific survival; TTR, time to recurrence; EGFR, epidermal growth factor receptor. 
prognostic value than that of mSEPT9 alone. The level of mSEPT9 was related to dMMR, mutations in TP53, BRAF, and PIK3CA, and was an effective biomarker for the prognosis of patients with CRC.

Keywords: colorectal cancer, mSEPT9, prognosis, dMMR, genetic mutation

\section{INTRODUCTION}

Colorectal cancer (CRC) is a common malignancy of the digestive tract. According to the GLOBOCAN data in 2018, with 1,800,000 cases, CRC ranked the third highest incidence rate among all malignancies detected worldwide. However, with about 880,000 deaths, CRC ranks the second in mortality rate for all malignant tumors globally (Bray et al., 2018). In recent years, screening methods such as fecal occult blood test (FOBT), endoscopy, and so on have been used for the early diagnosis and detection of CRC. However, low compliance with doctors' advice remains a problem. Therefore, developing a simple and safe detection method with high specificity and sensitivity would significantly improve preliminary screening of CRCs and increase the compliance of patients.

Aberrant epigenetic changes, such as hypermethylation of $\mathrm{CpG}$ island, are the initiating events of colorectal tumorigenesis, and the gene hypermethylation status may serve as a useful biomarker for the screening of CRC (Rex et al., 2017; Danese et al., 2019). Septin 9 is a family of genes encoding conserved skeleton proteins with GTPase activity (Nagata et al., 2003). Detection of the septin9 gene and protein in CRC confirmed their anticancer role in the occurrence and development of CRC. The hypermethylation of CpG island in the promoter of Septin9 can inhibit its normal gene expression and thus suppress the anticancer function (Toth et al., 2011). The detection of mSEPT9 in peripheral blood has been gradually adopted for the screening of CRC around the world (Sun et al., 2019). Moreover, our previous study has showed higher specificity and sensitivity of mSEPT9 detection in peripheral blood than that of FOBT (US Preventive Services Task Force et al., 2016).

Endoscopy is considered the best method of CRC screening, although it cannot effectively increase the diagnosis rate of CRC because of poor compliance by patients. In this study, we first focused on understanding whether mSEPT9 detection in peripheral blood can improve compliance to endoscopy and be used as an effective method for CRC screening. Further, although the treatment modalities for cancer, including chemotherapy, surgery, targeted therapy, and immunotherapy have significantly improved, the mortality rate of CRC remains high due to the complexity of its pathogenesis. CRC is caused by accumulation and interaction of multiple gene mutations and epigenetic changes. The specific mechanisms predominantly include chromosomal instability (CIN), microsatellite instability (MSI), and CpG island methylation phenotype (CIMP) (Simons et al., 2013; Overman et al., 2018). Mutations in KRAS, NRAS, BRAF, and PIK3CA mainly occur in CIN and are partially associated with CIMP. There is widely recognized molecular typing of CRC, although the correlation between various pathogeneses remains unclear, and the complex relationship among different pathogeneses of CRC increases the difficulty of individual therapy (Guinney et al., 2015; Alwers et al., 2019; Stintzing et al., 2019). Thus, it is of great clinical significance to explore effective molecular indicators for evaluation of molecular typing and prognosis of CRC. Cetuximab, a chimeric monoclonal antibody targeting the epidermal growth factor receptor (EGFR), has been used as targeted adjuvant chemotherapy of patients with CRC harboring wild-type KRAS, NRAS, BRAF, and PIK3CA genotypes. However, the influence of CIMP on the drug efficacy is still unclear. At present, studies about mSEPT9 mainly focus on screening, diagnosis, prognosis, and correlation analysis with histopathology, and Shen $\mathrm{N}$ et al. reported that the hypermethylation of SEPT9 associated with worse overall survival (OS) and disease-free survival (DFS) (Shen et al., 2019), but there were no systematic reports about the relationship between mSEPT9 with specific molecular characteristics of CRC and the prognosis value of adjuvant chemotherapy or recurrence. Our previous study showed that mSEPT9 is related to the TNM stage, Dukes stage, and dMMR status of CRC (Sun et al., 2019). Thus, second, we have focused on the correlation between mSEPT9 and clinicopathological features, including routine histopathology, status of TP53 mutations, expression of mismatch repair (MMR) proteins, and KRAS, NRAS, BRAF, and PIK3CA genotypes. Third, the relation of these biomarkers with cancer-specific survival (CSS) and time to recurrence (TTR) in CRC patients treated with adjuvant chemotherapy was also studied. Study about mSEPT9 may provide newer molecular indicators for early diagnosis, treatment, prognosis evaluation, and recurrence monitoring of patients with CRC.

\section{MATERIALS AND METHODS}

\section{Study Population and Design}

For screening of CRC, 7759 individuals in Tianjin aged $>40$ years participated in the survey. The screening was carried out by a questionnaire survey, followed by endoscopy, and detection of FOBT and mSEPT9 in the peripheral blood. The questionnaire included basic information of residents, history of intestinal diseases, family history of CRC, and so on. These points were in line with the following positive prompts for high-risk populations: 1) history of CRC or adenoma; 2) first-degree relatives with a history of CRC; 3) coexistence of two or more of the following history: chronic diarrhea, chronic constipation, mucus bloody stool, chronic appendicitis or appendectomy, unhealthy life, and chronic choledochocystitis or cholecystectomy; and 4) have a direct relative with familial adenomatous polyposis and hereditary nonpolyposis CRC (HNPCC). Further, people with a high risk of CRC were randomly divided into three groups, viz. high-risk, high-risk + FOBT, and high-risk + mSEPT9. Each participant was suggested to undergo an endoscopy examination, but the subjects could independently choose not to accept the endoscopy. The number of subjects accepting the endoscopy examination among the positive and 


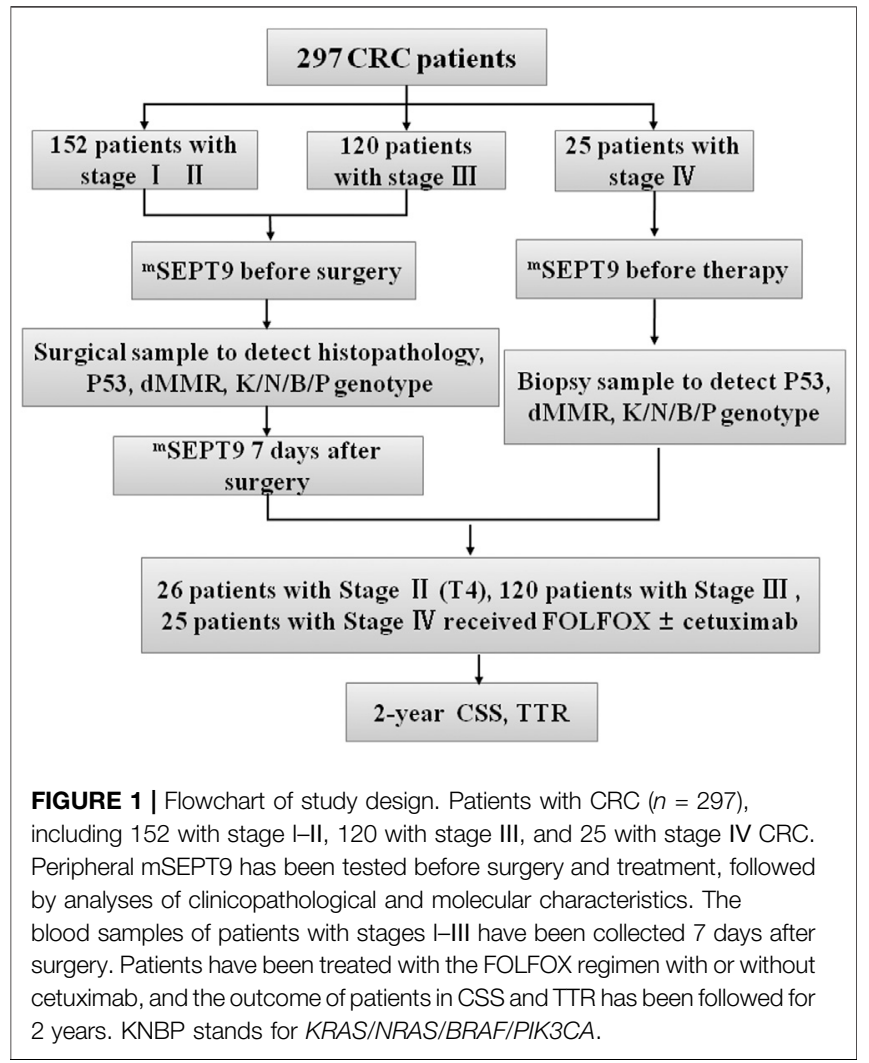

negative subjects of FOBT and mSEPT9 was counted to compare the compliance of the endoscopy. Furthermore, the sensitivity and specificity between FOBT and mSEPT9 were evaluated.

For evaluating the prognosis of MSEPT9 in a peripheral blood in training study, a database of patients who underwent the endoscopy examination and surgical treatment at the Tianjin Union Medical Center between January 2018 and April 2020 was retrospectively studied. The diagnosis and treatment programs were based on the guidelines of the National Comprehensive Cancer Network (NCCN) and Chinese Society of Clinical Oncology (CSCO) (Seal et al., 2014). Our study was performed according to the principles of the Declaration of Helsinki and was approved by the Research Ethics Committee of the Tianjin Union Medical Center, China. All the patients provided written informed consent for a peripheral blood test and use of their paraffin-embedded CRC tissues for genetic analyses. The specific study protocols are listed in Figure $\mathbf{1 .}$

The 297 CRC patients who were diagnosed by endoscopy included 272 patients with stages I-III and 25 patients with stage IV disease, whose blood samples were collected before radical operation and medical treatment, respectively. The tissue samples of 297 patients with stage I-IV CRC were also collected, and clinicopathological and molecular characteristics were analyzed. The blood samples of 272 patients with stage I-III CRC were collected 7 days after surgery. Patients with radical resection (stages I-III) were recruited to study the feasibility of assessing the levels of mSEPT9 DNA in plasma for recurrence evaluation and compared with those of carcinoembryonic antigen (CEA). Patients with wild-type KRAS, NRAS, BRAF, and PIK3CA genotypes were treated using the FOLFOX regimen [folinic acid (leucovorin calcium), fluorouracil, and oxaliplatin] and cetuximab, while those with mutated KRAS, NRAS, BRAF, and PIK3CA genotypes were treated with FOLFOX alone. The final diagnosis of recurrence was drawn based on the results of the endoscopy and postoperative pathological examination. Here, the diagnosis of recurrence included local recurrence and distant metastasis. The results of FOBT and CEA were directly collected from the clinical laboratory. The outcomes of all the patients in CSS and TTR were followed for 24 months. In a validation study, the clinicopathological data of an additional 226 patients with CRCs in the Tianjin Union Medical Center from December 1, 2018 to March 31, 2019 were collected to evaluate the prognostic value of mSEPT9. The outcome of all the patients in CSS and TTR was followed for 24 months.

\section{Sample Collection and Storage}

The peripheral blood samples $(10 \mathrm{ml})$ were collected in test tubes containing $\mathrm{K}_{2}$ EDTA anticoagulant (BD Biosciences, NJ, United States). Plasma samples $(3.5 \mathrm{ml})$ were collected upon centrifugation and stored under $-20^{\circ} \mathrm{C}$.

\section{Quantification of Methylated Septin9}

An improved SEPT9 gene methylation assay (Epigenomics AG for Epi proColon 2.0) was used for the screening of CRC in our study. DNA was extracted from plasma samples using the plasma processing kit (BioChain Science and Technology, Inc., Beijing). The DNA was then incubated with bisulfite, and methylated target sequences in the bisulfite-converted DNA template were amplified using real-time PCR. The methylation of SEPT9 in plasma samples was measured using an ABI7500 fluorescent PCR instrument. For $\mathrm{Ct}$ values $\leq 41$, the result was considered positive, whereas for Ct values $\geq 41$, the result was considered negative.

\section{Fecal Occult Blood Test}

An immune colloidal gold technique was utilized for the detection of fecal occult blood (FOB), and monoclonal antibodies were used to specifically target human hemoglobin (HB) in feces samples. The reaction line (T) on the cellulose nitrate membrane was coated with an anti-HB1 monoclonal antibody, and the control line (C) was coated with a sheep anti-mouse polyclonal antibody. When detected, the human hemoglobin in the sample could bind to the colloidal gold-antibody coated at the front of the reagent to form an immune complex. As the chromatographic complex moves along the membrane band, if it is a positive sample, it can agglutinate to form a color band on the reaction line (T) and the control line (C), respectively. If it is a negative sample, it will only form a color band on the control line (C). The lowest detectable level of hemoglobin was $0.2 \mu \mathrm{g} / \mathrm{ml}$, ranging from $0.2 \mu \mathrm{g} / \mathrm{ml}$ to $2000 \mu \mathrm{g} /$ $\mathrm{ml}$. For sample extraction, a $10-50 \mathrm{mg}$ sample was taken from six different parts of stool with a stool bar and mixed well in $0.5 \mathrm{ml}$ buffer solution for detection.

\section{Immunohistochemical Staining}

Sections with $4 \mu \mathrm{m}$ thickness of the CRC tissue samples were subjected to immunohistochemical analysis to detect the 
expression of mismatch repair proteins MLH1, MSH2, MSH6, PMS2, and p53. The MMR proteins expressed in normal intestinal epithelial cells, lymphocytes, smooth muscle cells, and vascular endothelial cells were used as the internal control. For a single mismatch repair protein, when the inner control cell nucleus was positive and cancer cell was negative (no nuclear staining), the protein expression was absent. In general, the absence of one or more MMR proteins in the tumor cells can be interpreted as MMR deficient (dMMR), and it corresponds to microsatellite instability-high (MSI-H) status. Whereas no deficiency of MMR proteins in tumor cells can be interpreted as MMR proficient (pMMR) and corresponds to microsatellite instability-low (MSI-L) or microsatellite stability (MSS) status. The absence of any of the proteins was interpreted as dMMR (Luchini et al., 2019). Each result was confirmed by two experienced pathologists.

\section{Mutation Analysis of KRAS/NRAS/BRAF/ PIK3CA}

The DNA from paraffin-embedded samples was extracted using the Qiagen extraction kit (Cat No.: 56404, Germany), and its genotype was determined using PCR analysis. We analyzed 12 somatic hot spot mutations in exons 2, 3, and 4 of the human KRAS gene, 3 somatic hot spot mutations in exons 2 and 3 of the human NRAS gene, V600E hot spot mutation in exon 15 of the human $B R A F$ gene, and 5 hot spot mutations in exons 9 and 20 of the human PIK3CA gene.

\section{Statistical Analyses}

All statistical analyses were performed using the SPSS version 25.0. ANOVA and $t$ tests were performed to compare the differences of mSEPT9 levels among different groups, and to analyze the association of $\mathrm{Ct}$ values of MSEPT9 with specific molecules of dMMR. The survival analysis was based on CSS and TTR. The CSS was defined as the time from CRC diagnosis to CRC-related death or end of follow-up. TTR was calculated as the period from surgery to either death from CRC, local recurrence, or distal metastasis. The influence of biomarkers on CSS and TTR was analyzed using the Kaplan-Meier curves and Wilcoxon test. Hazard ratios (HRs) and 95\% confidence intervals (CIs) for CRC-related deaths were estimated using Cox regression analysis. Multivariable adjusted models included the predefined potential prognostic factors, such as age at diagnosis, sex, tumor localization, and TNM stage. A two-sided $p$ value $<0.05$ was considered statistically significant.

\section{RESULTS}

\section{Detection of Methylated Septin9 Improves Compliance of Endoscopic Examination for Screening of Colorectal Cancer}

Of the 7759 participants, 3578 were men with a median age of 55 years, while 4181 were women with a median age of
56 years. Based on the results of the questionnaire survey, there were 882 individuals with a high risk of CRC. Further, 105 subjects belonged to the high-risk group according to criterion (1), 449 belonged to criterion (2), 367 belonged to criterion (3), and 41 belonged to criterion (4) (some subjects meet two criteria). These criteria have been listed in the study population and design. These 882 individuals were randomly divided into the three groups, viz. high-risk, high-risk + FOBT, and high-risk + mSEPT9, and each group contained 294 subjects. The percentage of patients who volunteered individuals for endoscopy examination in the high-risk group, positive FOBT group, and positive mSEPT9 group were $23 \%, 42 \%$, and $96 \%$, respectively, which may be associated with the high sensitivity and specificity of the peripheral mSEPT9 test in the early screening of CRCs. The positive test result of mSEPT9 contributed to higher compliance of endoscopy examination than that of positive FOBT. The rate of endoscopy examination in the negative mSEPT9 group was $15 \%$ (Table 1). There were 55 patients with CRC confirmed by an endoscope biopsy. Of these, 31 were men with a median age of 64 years, and 24 were women with a median age of 67 years. Furthermore, 25 cases were detected in the colon and 30 in the rectum. The sensitivity and specificity of $\mathrm{mSEPT} 9$ were $73 \%$ and $98 \%$, respectively, which were significantly higher than the respective $55 \%$ and $86 \%$ values for FOBT (Table 2). Thus, based on the results obtained, detection of peripheral blood mSEPT9 is an effective way to address the poor compliance of endoscopy examination, and the sensitivity and specificity of mSEPT9 detection are better than the clinical routine FOBT detection.

\section{Correlation of Methylated Septin9 With Clinicopathological and Molecular Characteristics of Colorectal Cancer}

The clinical characteristics of 297 CRC patients who underwent the procedure for detection of peripheral mSEPT9 are summarized in Table 3. The correlation analysis for the result of peripheral mSEPT9 with clinicopathological and molecular characteristics is summarized in Table 4. The mSEPT9 was not significantly associated with the location of CRC. With the increase in the TNM stage, the Ct values of mSEPT9 decreased, indicating an increased release of mSEPT9 protein by tumor cells into the peripheral blood. As per the TNM stage, the Ct value of mSEPT9 was highest in stage I, lowest in stage IV, and statistically insignificant in stages II and III. There were 59 cases of dMMR, which contributed to $19.9 \%$ of 297 cases with CRC. Moreover, the positive rate of mSEPT9 in stage II was higher than that in stage III, which may be due to occurrence of dMMR in stage II, and that the positive rate of mSEPT9 in dMMR was higher than that in pMMR. Furthermore, the negative expression of MLH-1 mainly occurred in positive mSEPT9 samples, while positive expression had a higher possibility of occurrence in negative mSEPT9 samples. These results further validated the association between mSEPT9 and dMMR (Figure 2A a and b). Additionally, the MLH-1 expression was highest in patients with positive 
TABLE 1 | Function of FOBT and mSEPT9 in endoscope compliance.

\begin{tabular}{|c|c|c|c|}
\hline & No. of patients & Endoscopy examination & $p$ \\
\hline High-risk & 294 & $68(23 \%)$ & \\
\hline High-risk + FOBT negative & 242 & $39(16 \%)$ & $0.73^{\mathrm{a}}$ \\
\hline High-risk + FOBT positive & 52 & $22(42 \%)$ & $0.02^{\mathrm{a}}$ \\
\hline High-risk + mSEPT9 negative & 271 & $41(15 \%)$ & $0.81^{a}$ \\
\hline High-risk + mSEPT9 positive & 23 & 21 (96\%) & $<0.001^{a}, 0.01^{b}$ \\
\hline
\end{tabular}

${ }^{a} \mathrm{p}$ value for the comparison with the high-risk group.

${ }^{b} \mathrm{p}$ value for the comparison between the MSEPT9 positive group and FOBT positive group.

TABLE 2 | Sensitivity and specificity of mSEPT9 and FOBT detection in the screening of CRC for the high-risk population.

\begin{tabular}{|c|c|c|c|c|c|c|}
\hline & $\begin{array}{l}\text { Number of total } \\
\text { CRC patients }\end{array}$ & $\begin{array}{c}\text { Number of CRC patients with } \\
\text { positive SEPT9 or FOBT } \\
\text { result }\end{array}$ & $\begin{array}{l}\text { Sensitivity } \\
(95 \% \mathrm{Cl})\end{array}$ & $\begin{array}{c}\text { Number of total } \\
\text { healthy participants }\end{array}$ & $\begin{array}{c}\text { Number of healthy participants } \\
\text { with negative SEPT9 or FOBT } \\
\text { result }\end{array}$ & $\begin{array}{l}\text { Specificity } \\
(95 \% \text { Cl) }\end{array}$ \\
\hline mSEPT9 & 26 & 19 & $73 \%(62-81 \%)$ & 264 & 260 & 98\% (89-99\%) \\
\hline FOBT & 29 & 16 & $55 \%(44-65 \%)$ & 265 & 229 & $86 \%(81-90 \%)$ \\
\hline
\end{tabular}

TABLE 3 | Clinical characteristics of 297 patients with CRC.

\begin{tabular}{lc} 
Variable & No. of patients \\
\hline Age & \\
$<40$ years & 12 \\
$40-50$ years & 34 \\
$51-60$ years & 72 \\
$61-70$ years & 112 \\
$>70$ years & 67 \\
Gender & \\
Female & 127 \\
Male & 170 \\
History & \\
One first-degree relative with CRC & 30 \\
Intestinal adenoma or polyps & 59 \\
Chronic constipation & 105 \\
Chronic diarrhea & 82 \\
Inflammatory colon diseases & 37 \\
Mucus and bloody stool & 64 \\
Chronic appendicitis or appendectomy & 11 \\
Smoking & 113 \\
Drinking & 202 \\
Non & 42
\end{tabular}

mSEPT9 (Figure 2B). Of the 297 patients with CRC, 97 cases had the wild-type status of TP53, and 200 had mutant TP53. The Ct values of mSEPT9 in patients with TP53 missense mutation was less than that in those with wild-type TP53. Results of immunohistochemical staining showed that increased TP53 missense mutations occurred in the positive mSEPT9 group, indicating that hypermethylation of CpG island of SEPT9 may promote TP53 missense mutations in patients with CRC (Figure 2A $\mathrm{c}$ and $\mathrm{d}$ ). The mutation rate in KRAS/NRAS was 49.2\%, BRAF was 5.1\%, and PIK3CA was $2 \%$ in all these 297 patients with CRC. In the study of the correlation between mSEPT9 and KRAS/NRAS/BRAF/PIK3CA mutation genes, mSEPT9 was related to BRAF and PIK3CA gene mutations but not to KRAS and NRAS mutations (Table 4), suggesting that $\mathrm{mSEPT} 9$ may promote the downstream molecule mutations in the EGFR signaling pathway.

\section{Association Between Methylated Septin9 Levels, Specific Genotype Mutations, and Cancer-Specific Survival and Time to Recurrence}

In patients with stage I-IV CRC, there was no significant difference in the CSS between positive MSEPT9 and negative mSEPT9 before treatment. However, CRC patients whose mSEPT9 Ct value $\leq 37.5$ were associated with a shorter CSS than those with $>37.5 \mathrm{Ct}$ value (Table 5). Of the patients who received adjuvant chemotherapy, patients with $\mathrm{mSEPT} 9 \mathrm{Ct}$ value $\leq 37.5$ had worse CSS than those with Ct value $>37.5$ (Figure 3A), which was consistent with the validation study result (Figure 3B). There was no significant difference for CSS between dMMR and pMMR in CRC patients, and the prognosis between dMMR and pMMR was not affected by mSEPT9 expression levels (Ct value 337.5). Patients with KRAS/NRAS-mutated tumors were associated with a shorter CSS than those with the wild-type status, although the Ct value of mSEPT9 had no significant aggravation of CSS on patients with KRAS/NRAS-mutated tumors. The patients with BRAF-mutated tumors had shorter CSS than those with BRAF wild-type tumors, and those with a $\mathrm{Ct}$ value of $\mathrm{mSEPT} 9 \leq 37.5$ tumors harboring BRAF mutations had worse CSS than those with a Ct value of mSEPT9 $>37.5$ tumors harboring BRAF-mutated tumors. No significant difference was observed between patients with PIK3CA-mutated tumors and those with PIK3CA wild-type tumors, but the PIK3CA-mutated tumors with positive mSEPT9 (Ct value $\leq 37.5$ ) correlated with worse CSS than those with Ct value of mSEPT9 > 37.5 tumors harboring PIK3CA mutation. Further, in the analysis of relationship of patients' TTR and stage I-III CRC (Table 5), the Ct value of mSEPT9 $\leq 37.5$ was associated with shorter TTR than in those with the $\mathrm{Ct}$ value $>37.5$. Of the patients who received adjuvant chemotherapy, patients with $\mathrm{mSEPT} 9 \mathrm{Ct}$ 

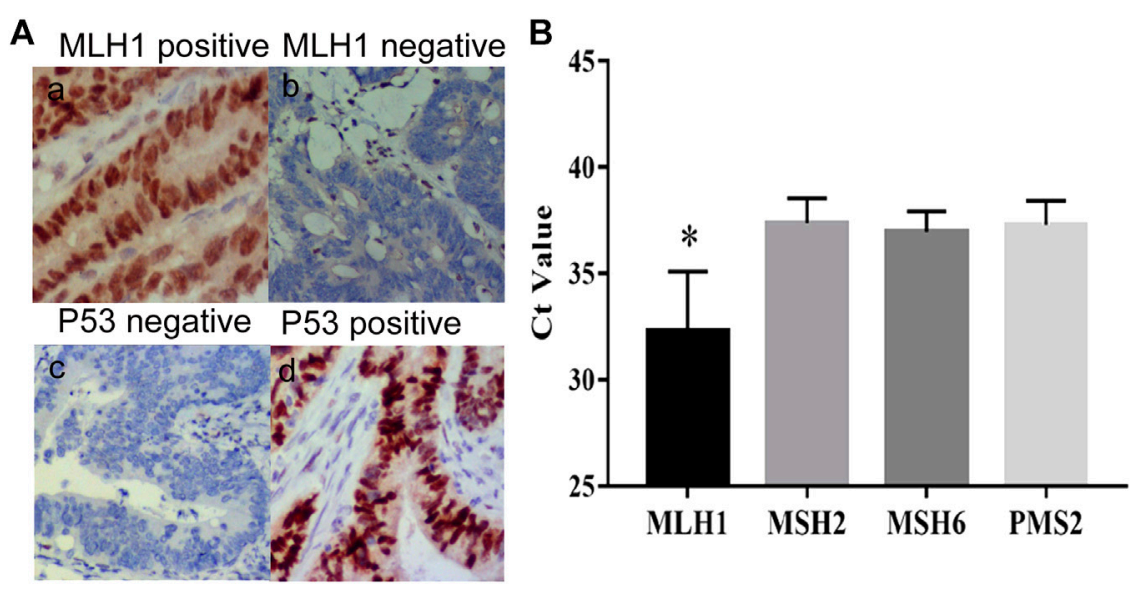

FIGURE 2 | Expression of MLH1 and P53. (A) (a) Positive expression of MLH1 (IHC, magnification $\times 200$ ). (b) Negative expression of MLH1 (IHC, magnification $\times$ 200). (c) Negative expression of P53 (IHC, magnification $\times 200$ ). (d) Positive expression of P53 (IHC, magnification $\times 200)$. (B) The Ct values of mSEPT9 with specific molecules of dMMR. The Ct value of MLH1 is the highest among the four specific molecules of dMMR $\left({ }^{*} p<0.01\right)$.

value $\leq 37.5$ had worse TTR than in those with the $\mathrm{Ct}$ value $>37.5$ (Figure 3C), which was consistent with the validation study result (Figure 3D). Moreover, the Ct value of mSEPT9 did not affect the TTR related to dMMR and KRAS/NRAS mutations, although the status of mSEPT9 (Ct value $\leq 37.5$ ) reduced the TTR of patients with BRAF or PIK3CA mutations. This suggested that mSEPT9 could affect the mutations in members downstream to the EGFR signaling pathway and further affect the treatment outcome with adjuvant chemotherapy.

\section{The Prognostic Effect of Methylated Septin9 on Specific Genotype Mutation in Different Colorectal Cancer Stages}

Patients with stage I-III CRC with mSEPT9 Ct value $\leq 37.5$ had significantly worse CSS than those with mSEPT9 Ct value >37.5. There was no difference in CSS in patients with stage IV CRC. Patients with stage I-II CRC with the dMMR status was independently associated with better CSS but not in those with stage III and stage IV CRC. Moreover, dMMR with positive mSEPT9 (Ct value $\leq 37.5$ ) in stage III CRC was associated with worse CSS than that with dMMR. No differences in CSS were observed in patients with stage I-II CRC irrespective of mutated or wild-type status of KRAS/NRAS/BRAF/PIK3CA, although significant differences in CSS was observed for stage III and IV patients with mutated and wild-type tumors. Additionally, the BRAF- or PIK3CA-mutated tumors with positive mSEPT9 (Ct value $\leq 37.5$ ) had shorter CSS in stages III and IV than the tumors with $\mathrm{Ct}$ value of $\mathrm{mSEPT} 9>37.5$ (Table 6).

\section{The Role of Methylated Septin9 in the Evaluation of Surgical Effectiveness in Stage I-III Colorectal Cancer}

To study the prognostic value of mSEPT9 in a surgical effectiveness and TTR in patients with stage I-III CRC, we tested peripheral mSEPT9 levels 7 days after surgery and simultaneously collected clinical regular examination results for CEA. The recurrence rate in patients with positive mSEPT9 was higher than those with positive CEA. Furthermore, the recurrence rate in patients with mSEPT9 combined with CEA was higher than in those with mSEPT9 alone, which indicated that $\mathrm{mSEPT} 9$ was a useful predictive marker for the evaluation of postsurgical prognosis of CRC (Table 7; Figure 3E).

\section{DISCUSSION}

Early diagnosis and treatment is the key to improve prognosis of CRC patients. Lofton-Day C. et al. detected the expression of methylated TMEFF2, NGFR, and SEPT9 genes in plasma samples of CRC patients and healthy controls using the reverse transcription polymerase chain reaction, and the results confirmed that the methylated SEPT9 gene was highly expressed in cancer tissues and the peripheral blood of CRC patients (Lofton-Day et al., 2008). The high sensitivity and specificity of mSEPT9 in peripheral blood makes it an ideal tool for the screening of CRC, which can be carried out using liquid biopsy technology molecular indicators for circulating tumor DNA (ctDNA). At present, FOBT and endoscopy are the predominant methods for early screening of patients with a high risk of CRC. However, given the poor sensitivity and specificity of FOBT, screening of CRC using FOBT cannot effectively improve the compliance for endoscopy. Our previous experiments showed that MSEPT9 was superior to FOBT in the specificity and sensitivity for early screening of CRC (Sun et al., 2019). Here, we analyzed whether the application of mSEPT9 could improve the compliance of endoscopy detection, and the results showed that the rate of endoscopy examination in patients with positive MSEPT9 was significantly higher than those with positive FOBT. 
TABLE 4 | Clinicopathological and molecular characteristics related to mSEPT9 in 297 cases of CRC.

Clinicopathological parameter

Localization

Colon

Rectosigmoid transition

Rectum

Invasive depth

$\begin{array}{ll}\mathrm{T} 1 & 19(6.4 \%)\end{array}$

T2 $60(20.2 \%)$

T3 $141(47.5 \%)$

T4 77 (25.9\%)

Lymph node metastasis

NO $\quad 152(51.2 \%)$

N1 $\quad 93(31.3 \%)$

N2 $\quad 52(17.5 \%)$

Distant metastasis

MO

M1

$272(91.6 \%)$

$25(8.4 \%)$

88.6

100

90.2

85.7

90.0

37.4

$\begin{array}{ll}36.6 & 0.097\end{array}$

$\begin{array}{lr}36.6 & 0.097 \\ 37.8 & 0.25\end{array}$

TNM stage

Stage I

Stage II

Stage III

Stage IV

Histopathological grade

High differentiation

High-middle/middle differentiation

Middle-low/low

Mucinous adenocarcinoma/signet-ring cell carcinoma

$24(8.1 \%)$

$128(43.1 \%)$

$120(40.4 \%)$

25 (8.4\%)

73.7
88.3
86.5
100

40.2

38.7

37.9

35.2

0.034

0.049

0.016

\section{Gross tumor volume}

0-10 $\mathrm{cm}^{3}$

$>10 \mathrm{~cm}^{3}$

$155(52.2 \%)$

$142(47.8 \%)$

104 (35.0\%)
59 (19.9\%)
$97(32.7 \%)$
37 (12.5\%)

93.3

83.1

90.7

86.5

75
93.0

86.7

100

90.1

89.2
88.5

38.1

36.6

37.4

0.11

0.082

\section{Morphology}

Protruded type

Ulcerative type

Mixed type

$120(40.4 \%)$

89.2

87.1

92.3

38.6

36.3

0.012

TP53 status

Wild-type

Mutated

97 (32.7\%)

MMR status

dMMR

pMMR

KRAS/NRAS status

Wild-type

$146(49.2 \%)$

BRAF status

Wild-type

Mutated

$282(94.9 \%)$

\section{PIK3CA status}

Wild-type

Mutated
89.3

100
$126(42.4 \%)$

$51(17.2 \%)$

92.1

84.3

$200(67.3 \%)$

85.6

91.5

39.

36.7

0.032

\section{9 (19.9\%)}

238 (80.1\%)

98.3

87.4

35.8

37.9

0.040

151 (50.8\%)

90.1

89.0

37.7

37.3

0.18

15 (5.1\%)

89.0

100

37.6

35.4

0.047
37.6

34.9
0.26

0.098

6 (2.0\%)

0.3
00


TABLE 5 | Univariate and multivariate Cox proportional hazard analysis of cancer-specific survival (stages I-IV) and time to recurrence (stages I-III).

Variable
Cancer-specific survival (CSS)

\begin{tabular}{lllll}
\multicolumn{2}{c}{ Univariate } & & & \multicolumn{2}{c}{ Multivariate } \\
\cline { 1 - 1 }$(95 \% \mathrm{Cl})$ & $p$ & & HR $(95 \% \mathrm{Cl})$ & $p$
\end{tabular}

Time to recurrence (TTR)

\begin{tabular}{lllll}
\hline \multicolumn{2}{c}{ Univariate } & & & \multicolumn{2}{c}{ Multivariate } \\
\cline { 1 - 1 }$(95 \% \mathrm{Cl})$ & $p$ & & HR $(95 \% \mathrm{Cl})$ & $p$
\end{tabular}

mSEPT9 Ct value

\begin{tabular}{|c|c|c|c|c|c|c|c|c|c|}
\hline$>37.5$ & 177 & 1 (ref) & & 1 (ref) & & 1 (ref) & & 1 (ref) & \\
\hline$\leq 37.5$ & 120 & $2.52(1.75-3.32)$ & 0.003 & $2.01(1.02-3.55)$ & 0.005 & $2.27(1.58-3.83)$ & 0.004 & 1.98 (2.26-3.67) & 0.009 \\
\hline
\end{tabular}

mSEPT9 status

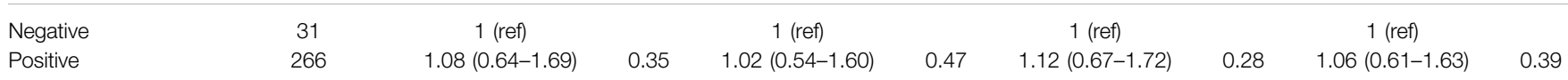

\section{TNM stage}

Stage I

Stage II

Stage III

Stage IV

24
128
120
25

1 (ref)
$1.73(1.20-2.49)$
$3.08(2.14-4.42)$
$5.90(4.34-9.41)$

0.02
0.0005
0.0001

0.045
0.001
0.0003

$\begin{array}{cc}1 \text { (ref) } & \\ 1.82(1.41-2.66) & 0.01 \\ 2.91(2.02-4.15) & 0.0008 \\ 5.05(3.51-8.22) & 0.0002\end{array}$

1 (ref)
$(0.98-2.8$

$1.59(0.98-2.22)$

2.65 (2.06-3.67)

$4.22(2.67-7.38)$
0.032 0.002

MMR status

dMMR
dMMR + mSEPT9
PMMR
pMMR + mSEPT9

$\begin{array}{ccc}59 & 1 \text { (ref) } & \\ 32 & 0.91(0.59-1.63) & 0.42 \\ 238 & 1.15(0.49-1.71) & 0.25 \\ 99 & 1.07(0.60-1.63) & 0.38\end{array}$

$\begin{array}{cc}1 \text { (ref) } & \\ 0.97(0.61-1.66) & 0.50 \\ 1.08(0.67-1.69) & 0.36 \\ 1.01(0.54-1.58) & 0.40\end{array}$

1 (ref)

$0.95(0.64-1.70)$

$1.21(0.82-1.99)$

$1.09(0.69-1.73)$

0.47
0.18
0.32

1 (ref)

$0.99(0.64-1.70) \quad 0.58$

$1.16(0.70-1.75) \quad 0.22$

$1.05(0.61-1.63) \quad 0.42$

KRAS/NRAS status

\begin{tabular}{|c|c|c|c|c|c|c|c|c|c|}
\hline Wild-type & 151 & 1 (ref) & & 1 (ref) & & 1 (ref) & & 1 (ref) & \\
\hline Wild + mSEPT9 & 72 & $1.09(0.61-1.68)$ & 0.35 & $1.03(0.52-1.57)$ & 0.40 & $1.13(0.80-1.76)$ & 0.26 & $1.02(0.55-1.59)$ & 0.43 \\
\hline Mutated & 146 & 2.78 (1.93-3.63) & 0.002 & $2.56(1.73-3.42)$ & 0.005 & 2.92 (2.01-3.98) & 0.001 & $2.76(1.89-3.77)$ & 0.006 \\
\hline Mutated + mSEPT9 & 86 & $1.17(0.72-1.78)$ & 0.20 & $1.28(0.85-1.86)$ & 0.14 & $1.11(0.69-1.75)$ & 0.42 & $1.01(0.57-1.60)$ & 0.42 \\
\hline \multicolumn{10}{|l|}{$B R A F$ status } \\
\hline Wild-type & 282 & 1 (ref) & & 1 (ref) & & 1 (ref) & & 1 (ref) & \\
\hline Wild + mSEPT9 & 139 & $1.13(0.63-1.72)$ & 0.25 & 1.07 (0.55-1.68) & 0.33 & $1.21(0.74-1.83)$ & 0.13 & $1.15(0.68-1.79)$ & 0.22 \\
\hline Mutated & 15 & 2.67 (1.86-3.49) & 0.002 & $2.46(1.61-3.22)$ & 0.004 & $2.72(1.91-3.68)$ & 0.001 & 2.69 (1.85-3.51) & 0.002 \\
\hline Mutated + mSEPT9 & 13 & $2.62(1.85-3.52)$ & 0.002 & $2.38(1.49-3.01)$ & 0.005 & 2.69 (1.88-3.92) & 0.002 & $2.51(1.71-3.50)$ & 0.003 \\
\hline \multicolumn{10}{|l|}{ PIKЗCA status } \\
\hline Wild-type & 291 & 1 (ref) & & 1 (ref) & & 1 (ref) & & 1 (ref) & \\
\hline Wild + mSEPT9 & 128 & $0.96(0.58-1.62)$ & 0.55 & $0.98(0.57-1.63)$ & 0.62 & $1.05(0.60-1.71)$ & 0.30 & 1.02 (0.59-1.67) & 0.37 \\
\hline Mutated & 6 & $1.06(0.66-1.75)$ & 0.40 & $1.02(0.58-1.65)$ & 0.44 & $1.08(0.65-1.77)$ & 0.37 & $1.03(0.69-1.74)$ & 0.40 \\
\hline Mutated + mSEPT9 & 5 & $1.51(1.02-2.29)$ & 0.041 & $1.32(0.79-2.01)$ & 0.055 & $1.58(1.10-2.28)$ & 0.038 & 1.53 (1.06-2.33) & 0.049 \\
\hline
\end{tabular}

Owing to the heterogeneity of the tumor reflected in multiple pathway-related molecular alterations, there is considerable stage independent variability in clinicopathological performance (Kim et al., 2013; Sinicrope et al., 2017; Guo et al., 2019). The DNA repair system regulates the pathogenesis of CRC, and the loss or reduction in function of dMMR cells to repair mismatched bases leads to the formation of MSI, instability of the MSIrelated genome, and an increase in tumor susceptibility (Dienstmann et al., 2019). Most of the microsatellite sequences are located in the coding region of genes involved in oncogenesis and development. The absence of the MMR system can inhibit the tumor suppressor genes and activate oncogenes (Lochhead et al., 2013). The frequency of chromosomal aberrations in CRC patients is 50-85\%. Chromosomal instability can lead to the activation of oncogenes, such as the Ras, and inactivation of tumor suppressor genes, such as APC and TP53, which usually reduce the duration of overall survival and progression free survival and cause adverse prognosis after treatment with 5-fluorouracil (Liu et al., 2015; Aghagolzadeh and Radpour, 2016). CIMP is closely related to many molecular features, including MSI, epigenetic silencing of mismatch repair gene $M L H 1$, and mutations in TP53, BRAF, and KRAS (Dawson et al., 2014; Koelzer et al., 2015; Vedeld et al., 2017). Moreover, CIMP is also associated with few clinicopathological features, including tumor localization, gender, age, tumor type, differentiation, and so on. A few 

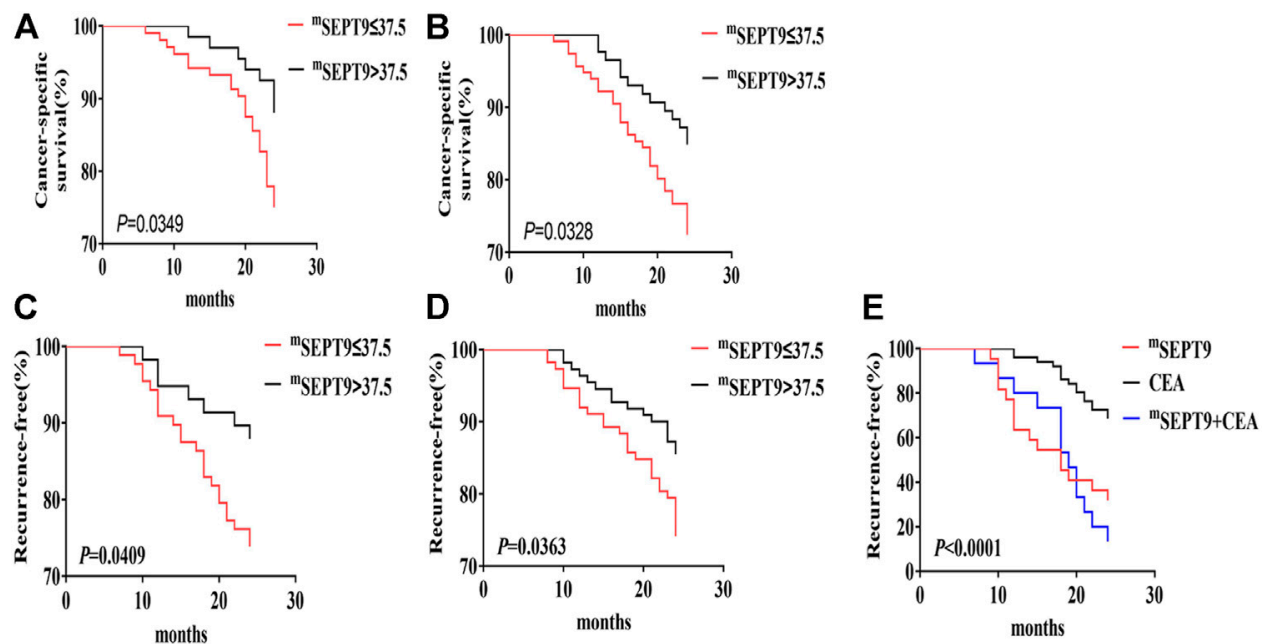

FIGURE 3 |CSS and TTR analysis of mSEPT9. (A) Kaplan-Meier curves of CSS in the overall cohort based on the Ct value of mSEPT9 before surgery or treatment with adjuvant chemotherapy. (B) The validation study of Kaplan-Meier curves of CSS in the overall cohort based on the Ct value of mSEPT9 before surgery or treatment with adjuvant chemotherapy. (C) Kaplan-Meier curves of TTR in patients with stage I-III CRC, based on the Ct value of mSEPT9 before surgery or treatment with adjuvant chemotherapy. (D) The validation study of Kaplan-Meier curves of TTR in patients with stage I-III CRC, based on the Ct value of mSEPT9 before surgery or treatment with adjuvant chemotherapy. (E) Kaplan-Meier curves of TTR in patients with stages I-III, based on the Ct value of mSEPT9, CEA, and combination of mSEPT9 and CEA measured 7 days after surgery.

TABLE 6 | Cox analysis of prognostic variables for CSS in colorectal patients from stage I to stage IV.

\begin{tabular}{|c|c|c|c|c|c|c|}
\hline \multirow[t]{2}{*}{ Variable } & \multicolumn{2}{|c|}{ Stages I-II } & \multicolumn{2}{|c|}{ Stage III } & \multicolumn{2}{|c|}{ Stage IV } \\
\hline & HR (95\% Cl) & $p$ & HR (95\% Cl) & $p$ & HR (95\% Cl) & $p$ \\
\hline \multicolumn{7}{|l|}{ mSEPT9 Ct value } \\
\hline$>37.5$ & $1.57(0.74-3.36)$ & 0.28 & $1.42(0.65-3.11)$ & 0.44 & $1.21(0.41-3.05)$ & 0.66 \\
\hline$\leq 37.5$ & $2.26(1.19-4.29)$ & 0.01 & $2.17(1.08-4.21)$ & 0.04 & $2.01(0.81-3.98)$ & 0.13 \\
\hline $\mathrm{dMMR}$ & $0.79(0.71-0.92)$ & 0.02 & $1.34(0.55-3.09)$ & 0.51 & $1.25(0.44-3.10)$ & 0.58 \\
\hline $\mathrm{dMMR}+$ mSEPT9 & $1.46(0.62-3.13)$ & 0.40 & $2.20(1.10-4.22)$ & 0.03 & $1.59(0.75-3.41)$ & 0.32 \\
\hline KRAS/NRAS mutated & $1.98(0.76-3.85)$ & 0.07 & $2.41(1.43-4.52)$ & 0.01 & $4.08(1.62-11.30)$ & 0.001 \\
\hline KRAS/NRAS mutated + mSEPT9 & $1.93(0.75-3.79)$ & 0.12 & $1.67(0.85-3.45)$ & 0.25 & $1.72(0.92-3.49)$ & 0.21 \\
\hline BRAF mutated & $1.89(0.71-3.81)$ & 0.16 & $2.22(1.15-4.28)$ & 0.04 & $2.53(1.39-5.02)$ & 0.005 \\
\hline BRAF mutated + mSEPT9 & $1.60(0.74-3.39)$ & 0.30 & $2.51(1.35-4.87)$ & 0.006 & $2.88(1.49-8.12)$ & 0.004 \\
\hline PIKЗCA mutated & $1.73(0.59-3.64)$ & 0.20 & $2.19(1.08-3.18)$ & 0.03 & $2.37(1.38-4.71)$ & 0.008 \\
\hline PIKЗCA mutated + mSEPT9 & $1.81(0.64-3.73)$ & 0.18 & $2.72(1.41-7.29)$ & 0.004 & $3.12(1.55-9.25)$ & 0.002 \\
\hline
\end{tabular}

TABLE 7 | Comparison of prognosis for TTR between mSEPT9 and CEA after radical operation.

\begin{tabular}{|c|c|c|c|}
\hline & Positive number & Number of recurrence & Rate of recurrence (\%) \\
\hline mSEPT9 & 22 & 15 & 68.2 \\
\hline CEA & 51 & 16 & 31.4 \\
\hline mSEPT9 + CEA & 15 & 13 & 86.7 \\
\hline
\end{tabular}

studies have shown that left-sided colon cancer is mainly characterized by chromosome instability, abnormal activation of the growth factor signaling pathway and the Wnt signaling pathway, and well differentiation. Whereas, right-sided colon cancer is characterized by high microsatellite instability, high methylation of $\mathrm{CpG}$ island, mutations in key carcinogenic proteins, mutations in $B R A F$, and expression of oncoproteins in the serrated adenoma (Tejpar et al., 2017). The pathological phenotype of rightsided colon cancer is poor differentiation, high proportion of advanced disease, increased mucinous adenocarcinoma, and high propensity for complications and second primary 
intestinal tumor that associates with poor prognosis (Missiaglia et al., 2014; Sveen et al., 2019).

Further, a previous study observed differences in the positive rate of SEPT9 methylation in the peripheral blood of CRC patients with varied clinicopathological characteristics, and its positive rate is significantly related to the malignant biological behavior (Shen et al., 2019). Our previous studies showed that the methylation of SEPT9 is associated with the TNM stage, total tumor volume, and mismatch repair deficient status (Sun et al., 2019), suggesting that mSEPT9 in peripheral blood may be related with the pathological stage and the prognosis of patients. In this study, we examined mSEPT9, the status of TP53 and DNA mismatch repair, and mutations in KRAS, NRAS, BRAF, and PIK3CA and their association with clinicopathological characteristics. The results indicated that the level of mSEPT9 in the peripheral blood of CRC patients was associated with missense mutations in TP53. The mSEPT9 levels could induce loss of dMMR-specific gene expression, especially the MLH1 gene. Furthermore, the levels of mSEPT9 were related to the mutations in BRAF and PIK3CA, consistent with results of previous studies (Fu et al., 2012; Sun et al., 2014; Kang et al., 2015). Furthermore, the Ct values of mSEPT9 before surgery were found to be associated with the overall prognosis of CRC patients, and the prognosis of patients who received adjuvant chemotherapy. It might be of greater clinical significance to analyze the combined prognostic evaluation value of mSEPT9 and different molecular indicators. Results of our study showed that mSEPT9 aggravated the influence on the prognosis of CRC patients with dMMR of stage III and BRAF/PIK3CA gene mutation of stages III-IV. However, no correlation was observed between mSEPT9 and patients' gender, tumor location, and differentiation.

About $30 \%$ of patients with stage III CRCs are prone to recur despite the administration of adjuvant chemotherapy. It is necessary to use an effective strategy after surgery to evaluate its therapeutic effect and provide a timely assessment and adjustment of the therapy (Shi et al., 2013). Serum CEA is a common marker recommended for recurrence monitoring of CRC in the clinics. However, its sensitivity and specificity are suboptimal. Studies have shown that the levels of mSEPT9 in peripheral blood decrease or become negative after radical surgery of CRC and then again become positive for tumor recurrence (Song et al., 2018), which suggests that mSEPT9 may be a molecular marker for monitoring the recurrence and metastasis of CRC. Furthermore, we analyzed the relationship between the results of ISEPT9 and TTR 7 days after operation, which showed that patients with positive mSEPT9 were significantly associated with worse TTR than in those with negative mSEPT9. This indicated that MSEPT9 may be helpful to predict the effect and outcome of surgery. Moreover, the prediction of CRC residual lesions after surgery using mSEPT9 was better than that using CEA, and the combined detection using mSEPT9 and CEA could improve the accuracy of postoperative evaluation of CRC.

\section{CONCLUSION}

The association of mSEPT9 with clinicopathological and molecular characteristics suggests that mSEPT9 may serve as an effective biomarker for screening and assessing the prognosis of CRC. Further study of mSEPT9 could optimize the molecular subtypes and improve the individualized treatment of CRC. In future, we will increase the sample size and extend the follow-up time to clarify the clinicopathological efficacy of mSEPT9 detection. Targeted therapy against hypermethylation of $\mathrm{CpG}$ island would play an important role in the treatment of CRC.

\section{DATA AVAILABILITY STATEMENT}

The original contributions presented in the study are included in the article/Supplementary Material, and further inquiries can be directed to the corresponding author.

\section{ETHICS STATEMENT}

The studies involving human participants were reviewed and approved by the Tianjin Union Medical Center. The ethics committee waived the requirement of written informed consent for participation.

\section{AUTHOR CONTRIBUTIONS}

ShZ designed the study; collected, analyzed, and interpreted data; contributed to manuscript writing; and approved the manuscript before submission. JS, JX, and CS collected and analyzed data and approved the manuscript before submission. JS, JX, CS, MZ, and YL collected, analyzed, and interpreted data; contributed to manuscript writing; and approved the manuscript before submission. SiZ collected data, gave constructive comments on the manuscript, and approved the manuscript before submission.

\section{FUNDING}

This work was supported in part by grants from the National Natural Science Foundation of China (No. 81672426), and Foundation of committee on science and technology of Tianjin (17ZXMFSY00120 and 17YFZCSY00700), and Foundation of Tianjin Union Medical Center (2018YJZD005). The funders had no roles in the design of the study, data collection, analysis and interpretation, or decision to write and publish the work.

\section{ACKNOWLEDGMENTS}

We acknowledge editage service for the manuscript language edit. 


\section{REFERENCES}

Aghagolzadeh, P., and Radpour, R. (2016). New Trends in Molecular and Cellular Biomarker Discovery for Colorectal Cancer. World J. Gastroenterol. 22, 5678-5693. doi:10.3748/wjg.v22.i25.5678

Alwers, E., Jia, M., Kloor, M., Bläker, H., Brenner, H., and Hoffmeister, M. (2019). Associations between Molecular Classifications of Colorectal Cancer and Patient Survival: A Systematic Review. Clin. Gastroenterol. Hepatol. 17, 402-410. doi:10.1016/j.cgh.2017.12.038

Bray, F., Ferlay, J., Soerjomataram, I., Siegel, R. L., Torre, L. A., and Jemal, A. (2018). Global Cancer Statistics 2018: GLOBOCAN Estimates of Incidence and Mortality Worldwide for 36 Cancers in 185 Countries. CA: A Cancer J. Clin. 68, 394-424. doi:10.3322/caac.21492

Danese, E., Montagnana, M., and Lippi, G. (2019). Circulating Molecular Biomarkers for Screening or Early Diagnosis of Colorectal Cancer: Which Is Ready for Prime Time?. Ann. Transl. Med. 7, 610. doi:10.21037/atm.2019. 08.97

Dawson, H., Galván, J. A., Helbling, M., Muller, D.-E., Karamitopoulou, E., Koelzer, V. H., et al. (2014). Possible Role of Cdx2 in the Serrated Pathway of Colorectal Cancer Characterized by BRAF Mutation, High-Level CpG Island Methylator Phenotype and Mismatch Repair-Deficiency. Int. J. Cancer 134, 2342-2351. doi:10.1002/ijc.28564

Dienstmann, R., Villacampa, G., Sveen, A., Mason, M. J., Niedzwiecki, D., Nesbakken, A., et al. (2019). Relative Contribution of Clinicopathological Variables, Genomic Markers, Transcriptomic Subtyping and Microenvironment Features for Outcome Prediction in Stage II/III Colorectal Cancer. Ann. Oncol. 30, 1622-1629. doi:10.1093/annonc/mdz287

Fu, T., Pappou, E. P., Guzzetta, A. A., Jeschke, J., Kwak, R., Dave, P., et al. (2012). CpG Island Methylator Phenotype-Positive Tumors in the Absence of MLH1 Methylation Constitute a Distinct Subset of Duodenal Adenocarcinomas and Are Associated with Poor Prognosis. Clin. Cancer Res. 18, 4743-4752. doi:10. 1158/1078-0432.ccr-12-0707

Guinney, J., Dienstmann, R., Wang, X., de Reyniès, A., Schlicker, A., Soneson, C., et al. (2015). The Consensus Molecular Subtypes of Colorectal Cancer. Nat. Med. 21, 1350-1356. doi:10.1038/nm.3967

Guo, T. A., Wu, Y. C., Tan, C., Jin, Y. T., Sheng, W. Q., Cai, S. J., et al. (2019). Clinicopathologic Features and Prognostic Value of KRAS, NRAS and BRAF Mutations and DNA Mismatch Repair Status: A Single-center Retrospective Study of 1,834 Chinese Patients With Stage I-IV Colorectal Cancer. Int. J. Cancer 145, 1625-1634. doi:10.1002/ijc.32489

Kang, K. J., Min, B.-H., Ryu, K. J., Kim, K.-M., Chang, D. K., Kim, J. J., et al. (2015). The Role of the CpG Island Methylator Phenotype on Survival Outcome in Colon Cancer. Gut Liver 9, 202-207. doi:10.5009/gnl13352

Kim, J. H., Rhee, Y.-Y., Bae, J.-M., Kwon, H.-J., Cho, N.-Y., Kim, M. J., et al. (2013). Subsets of Microsatellite-Unstable Colorectal Cancers Exhibit Discordance between the CpG Island Methylator Phenotype and MLH1 Methylation Status. Mod. Pathol. 26, 1013-1022. doi:10.1038/modpathol. 2012.241

Koelzer, V. H., Dawson, H., Andersson, E., Karamitopoulou, E., Masucci, G. V., Lugli, A., et al. (2015). Active Immunosurveillance in the Tumor Microenvironment of Colorectal Cancer Is Associated with Low Frequency Tumor Budding and Improved Outcome. Transl. Res. 166, 207-217. doi:10. 1016/j.trsl.2015.02.008

Liu, Y., Luan, L., and Wang, X. (2015). A Randomized Phase II Clinical Study of Combining Panitumumab and Bevacizumab, Plus Irinotecan, 5fluorouracil, and Leucovorin (FOLFIRI) Compared with FOLFIRI Alone as Second-Line Treatment for Patients with Metastatic Colorectal Cancer and KRAS Mutation. Onco Targets Ther. 8, 1061-1068. doi:10.2147/OTT. S81442

Lochhead, P., Kuchiba, A., Imamura, Y., Liao, X., Yamauchi, M., Nishihara, R., et al. (2013). Microsatellite Instability and BRAF Mutation Testing in Colorectal Cancer Prognostication. J. Natl. Cancer Inst. 105, 1151-1156. doi:10.1093/jnci/ djt173

Lofton-Day, C., Model, F., Devos, T., Tetzner, R., Distler, J., Schuster, M., et al. (2008). DNA Methylation Biomarkers for Blood-Based Colorectal Cancer Screening. Clin. Chem. 54, 414-423. doi:10.1373/clinchem.2007. 095992
Luchini, C., Bibeau, F., Ligtenberg, M. J. L., Singh, N., Nottegar, A., Bosse, T., et al. (2019). ESMO Recommendations on Microsatellite Instability Testing for Immunotherapy in Cancer, and its Relationship with PD-1/PD-L1 Expression and Tumour Mutational Burden: A Systematic Review-Based Approach. Ann. Oncol. 30, 1232-1243. doi:10.1093/annonc/mdz116

Missiaglia, E., Jacobs, B., D’ario, G., Di Narzo, A. F., Soneson, C., Budinska, E., et al. (2014). Distal and Proximal Colon Cancers Differ in Terms of Molecular, Pathological, and Clinical Features. Ann. Oncol. 25, 1995-2001. doi:10.1093/ annonc/mdu275

Nagata, K.-i., Kawajiri, A., Matsui, S., Takagishi, M., Shiromizu, T., Saitoh, N., et al. (2003). Filament Formation of MSF-A, a Mammalian Septin, in Human Mammary Epithelial Cells Depends on Interactions with Microtubules. J. Biol. Chem. 278, 18538-18543. doi:10.1074/jbc.m205246200

Overman, M. J., Adam, L., Raghav, K., Wang, J., Kee, B., Fogelman, D., et al. (2018). Phase II Study of Nab-Paclitaxel in Refractory Small Bowel Adenocarcinoma and CpG Island Methylator Phenotype (CIMP)-high Colorectal Cancer. Ann. Oncol. 29, 139-144. doi:10.1093/annonc/mdx688

Rex, D. K., Boland, R. C., Dominitz, J. A., Giardiello, F. M., Johnson, D. A., Kaltenbach, T., et al. (2017). Colorectal Cancer Screening: Recommendations for Physicians and Patients from the U.S. Multi-Society Task Force on Colorectal Cancer. Am. J. Gastroenterol. 112, 1016-1030. doi:10.1038/ajg. 2017.174

Seal, B. S., Sullivan, S. D., Ramsey, S. D., Shermock, K. M., Ren, J., Kreilick, C., et al. (2014). Systemic Therapy for Colorectal Cancer: Patterns of Chemotherapy and Biologic Therapy Use in Nationally Representative US Claims Database. BioDrugs 28, 229-236. doi:10.1007/s40259-013-0073-y

Shen, N., Wang, T., Li, D., Zhu, Y., Xie, H., and Lu, Y. (2019). Hypermethylation of the SEPT9 Gene Suggests Significantly Poor Prognosis in Cancer Patients: A Systematic Review and Meta-Analysis. Front. Genet. 10, 887. doi:10.3389/fgene. 2019.00887

Shi, Q., Andre, T., Grothey, A., Yothers, G., Hamilton, S. R., Bot, B. M., et al. (2013). Comparison of Outcomes after Fluorouracil-Based Adjuvant Therapy for Stages II and III Colon Cancer between 1978 to 1995 and 1996 to 2007: Evidence of Stage Migration from the ACCENT Database. J. Clin. Oncol. 31, 3656-3663. doi:10.1200/jco.2013.49.4344

Simons, C. C. J. M., Hughes, L. A. E., Smits, K. M., Khalid-De Bakker, C. A., de Bruïne, A. P., Carvalho, B., et al. (2013). A Novel Classification of Colorectal Tumors Based on Microsatellite Instability, the CpG Island Methylator Phenotype and Chromosomal Instability: Implications for Prognosis. Ann. Oncol. 24, 2048-2056. doi:10.1093/annonc/mdt076

Sinicrope, F. A., Shi, Q., Allegra, C. J., Smyrk, T. C., Thibodeau, S. N., Goldberg, R. M., et al. (2017). Association of DNA Mismatch Repair and Mutations in BRAF and KRAS with Survival after Recurrence in Stage III Colon Cancers. JAMA Oncol. 3, 472-480. doi:10.1001/jamaoncol.2016.5469

Song, L., Guo, S., Wang, J., Peng, X., Jia, J., Gong, Y., et al. (2018). The Blood mSEPT9 Is Capable of Assessing the Surgical Therapeutic Effect and the Prognosis of Colorectal Cancer. Biomarkers Med. 12, 961-973. doi:10.2217/ bmm-2018-0012

Stintzing, S., Wirapati, P., Lenz, H.-J., Neureiter, D., Fischer Von Weikersthal, L., Decker, T., et al. (2019). Consensus Molecular Subgroups (CMS) of Colorectal Cancer (CRC) and First-Line Efficacy of FOLFIRI Plus Cetuximab or Bevacizumab in the FIRE3 (AIO KRK-0306) Trial. Ann. Oncol. 30, 1796-1803. doi:10.1093/annonc/mdz387

Sun, J., Fei, F., Zhang, M., Li, Y., Zhang, X., Zhu, S., et al. (2019). The Role of (m) SEPT9 in Screening, Diagnosis, and Recurrence Monitoring of Colorectal Cancer. BMC Cancer 19, 450. doi:10.1186/s12885-019-5663-8

Sun, L., Guzzetta, A. A., Fu, T., Chen, J., Jeschke, J., Kwak, R., et al. (2014). CpG Island Methylator Phenotype and its Association with Malignancy in Sporadic Duodenal Adenomas. Epigenetics 9, 738-746. doi:10.4161/epi.28082

Sveen, A., Cremolini, C., and Dienstmann, R. (2019). Predictive Modeling in Colorectal Cancer: Time to Move Beyond Consensus Molecular Subtypes. Ann. Oncol. 30, 1682-1685. doi:10.1093/annonc/mdz412

Tejpar, S., Stintzing, S., Ciardiello, F., Tabernero, J., Van Cutsem, E., Beier, F., et al. (2017). Prognostic and Predictive Relevance of Primary Tumor Location in Patients with RAS Wild-Type Metastatic Colorectal Cancer. JAMA Oncol. 3, 194-201. doi:10.1001/jamaoncol.2016.3797

Tóth, K., Galamb, O., Spisák, S., Wichmann, B., Sipos, F., Valcz, G., et al. (2011). The Influence of Methylated Septin 9 Gene on RNA and Protein Level in 
Colorectal Cancer. Pathol. Oncol. Res. 17, 503-509. doi:10.1007/s12253-0109338-7

US Preventive Services Task Force Bibbins-Domingo, K., Grossman, D. C., Curry, S. J., Davidson, K. W., Epling, J. W., et al. (2016). Screening for Colorectal Cancer: US Preventive Services Task Force Recommendation Statement. JAMA 315, 2564-2575. doi:10.1001/jama.2016.5989

Vedeld, H. M., Merok, M., Jeanmougin, M., Danielsen, S. A., Honne, H., Presthus, G. K., et al. (2017). CpG Island Methylator Phenotype Identifies High Risk Patients Among Microsatellite stableBRAFmutated Colorectal Cancers. Int. J. Cancer 141, 967-976. doi:10.1002/ijc.30796
Conflict of Interest: The authors declare that the research was conducted in the absence of any commercial or financial relationships that could be construed as a potential conflict of interest.

Copyright $\odot 2021$ Sun, Xu, Sun, Zheng, Li, Zhu and Zhang. This is an open-access article distributed under the terms of the Creative Commons Attribution License (CC $B Y)$. The use, distribution or reproduction in other forums is permitted, provided the original author(s) and the copyright owner(s) are credited and that the original publication in this journal is cited, in accordance with accepted academic practice. No use, distribution or reproduction is permitted which does not comply with these terms. 